\title{
Analysis of Interference between UWB and ITS
}

\author{
Seho Park $\cdot$ Eun Cheol Kim $\cdot$ Jin Young Kim
}

\begin{abstract}
In this paper, we have analyzed the effect of interference between ultra-wideband(UWB) and intelligent transport systems(ITS). The maximum possible UWB emission power and minimum possible distance between UWB devices and ITS are found. In order to analyze the interference, we employ the Monte-Carlo(MC) method. We consider six situations, which are indoor office line-of-sight(LOS), indoor office non-line-of-sight(NLOS), indoor residential LOS, indoor residential NLOS, outdoor rural LOS, and outdoor rural NLOS environments. From the simulation results, it is confirmed that coexistence between UWB and ITS devices can be realized in accordance with the emission mask of $19.3 \mathrm{~dB}$ for indoor application or $19.3 \mathrm{~dB}$ for an image system. And in the outdoors, coexistence between UWB and ITS devices can be realized if the emission mask is at least $1.6 \mathrm{~dB}$ for vehicles' radar systems.
\end{abstract}

Key words : Interference Analysis, Intelligent Transport Systems(ITS), Monte-Carlo(MC), Ultra-wideband(UWB).

\section{Introduction}

Ultra-wideband(UWB) technology is one of the solutions for future data communication applications. The Federal Communication Commission(FCC) defines a radio system to be a UWB system if the fractional bandwidth or the $-10 \mathrm{~dB}$ bandwidth of the signal is greater than $20 \%$ or greater than $500 \mathrm{MHz}$, respectively ${ }^{[1]}$.

UWB systems are targeted at indoor environments providing high-speed communications, precision location and tracking, and short-range, wall-penetrating radar. UWB systems can generate interference with other radio communication systems because the UWB system reuses existing radio spectrum. The interference of a UWB system on an existing radio communication system depends on the overall characteristics of the concerned UWB system: transmitter power, modulation technique and density of UWB equipment. Therefore, the FCC has regulated the emission power of UWB systems and released regulation in $2002^{[1]}$. But the FCC's regulation was not adequate for some situations and communication systems. Therefore, the users of licensed bands worried about the impact on their existing service and strongly opposed the emission limit. For this reason, so many studies on the compatibility of UWB and existing radio communication systems have been advanced ${ }^{[2]}$. The coexistence with communication systems is the most important issue in order to use UWB devices commonly in the near future. The effect of UWB interference on a wireless local area network(WLAN), global system for mobile communication(GSM), and Bluetooth has already been analyzed ${ }^{[3] \sim[5]}$. The interference effect of a UWB system on GSM, WLAN, and Bluetooth is analyzed by Monte-Carlo(MC) simulation methodology. The MC method can address virtually all radio-interference scenarios. This flexibility is achieved by the way in which the parameters of the system are defined. It is possible to model even very complex situations by relatively simple elementary functions. Broadcasting systems, mobile systems, point-to-point systems and point-to-multipoint systems can be treated. The MC method also addresses other effects present in the radio environment such as receiver blocking and inter-modulation ${ }^{[6]}$. In this paper, the effect of UWB interference on intelligent transport systems(ITS) is analyzed and simulated.

This paper is organized as follows. In Section $\Pi$, UWB and ITS systems are overviewed. In Section III, the effect of interference between UWB devices and ITS devices is analyzed. Numerical results and simulation results are presented in Section IV. Finally, conclusion remarks are drawn in Section $\mathrm{V}$.

\section{UWB and ITS Overview}

\section{2-1 UWB}

Considered a recent breakthrough in broadband wireless technology, UWB is not a new invention, but it is has been researched since the $1960 \mathrm{~s}^{[7]}$. A traditional UWB transmitter operates by transmitting billions of pulses across a very wide spectrum of frequencies several $\mathrm{GHz}$ in bandwidth. The receiver then translates the pulses

Manuscript received August 25, 2009 ; revised October 20, 2009. (ID No. 20090825-036J)

Department of Wireless Communications Engineering, Kwangwoon University, Seoul, Korea. 
into data by listening for a familiar pulse sequence sent by the transmitter. UWB is defined as any radio technology having a spectrum that occupies a bandwidth greater than 20 percent of the center frequency or a bandwidth of at least $500 \mathrm{MHz}$.

The development of UWB has been considered for many years in laboratories, and basically, it has become standardized. There are two competing physical layer specifications available; one that is based on direct sequence(DS) UWB and the other that is based on multiband orthogonal frequency division multiplexing(MB OFDM). These two alternatives are currently under consideration by the IEEE 802.15 task group 3a.

With the regulation of UWB by the FCC, there was a debate over how much interference UWB would pose to existing radio services. The FCC approved the development of UWB on an unlicensed basis within a 3.1 $\sim 10.6 \mathrm{GHz}$ band in $2002^{[1]}$. The essence of this ruling

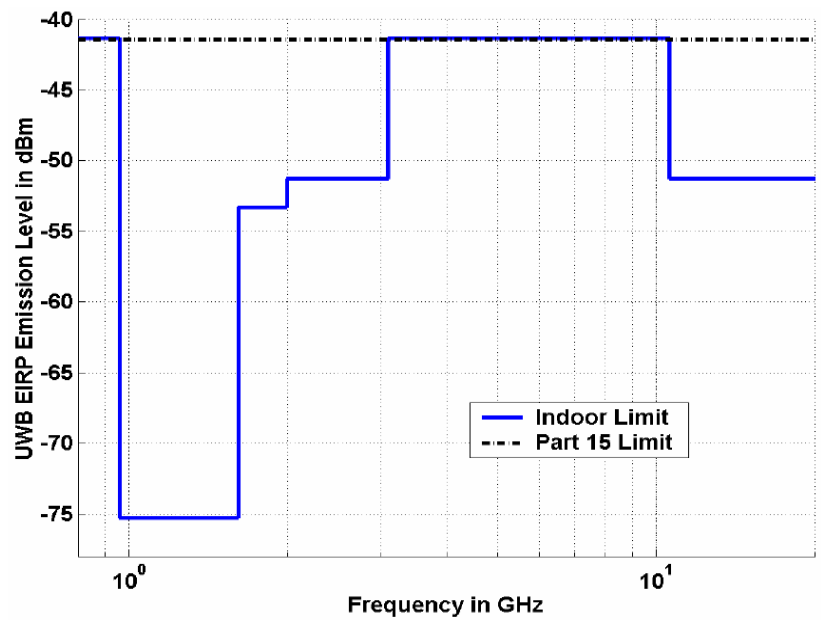

Fig. 1. Spectrum mask of UWB for indoor environments.

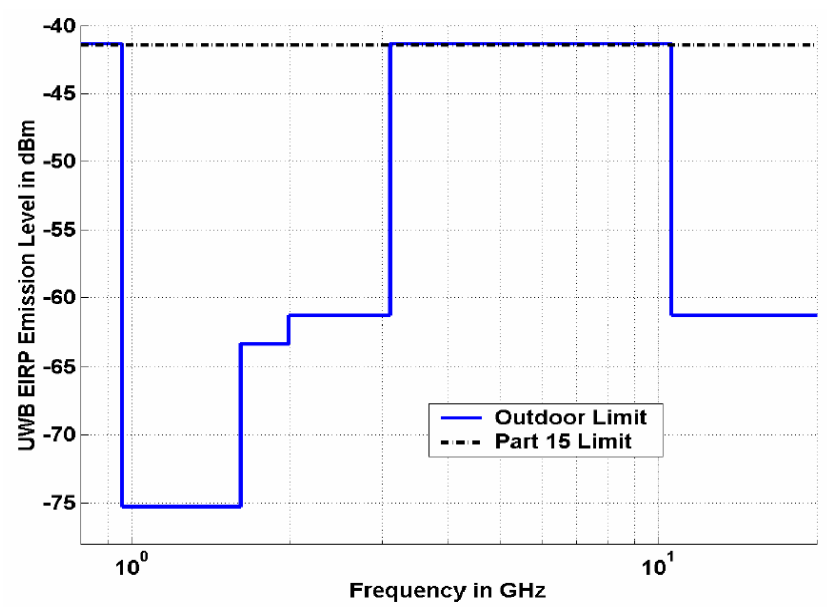

Fig. 2. Spectrum mask of UWB for outdoor environments. is to limit the power spectral density(PSD) measures in a $1 \mathrm{MHz}$ bandwidth at the output of an isotropic transmitting antenna to a spectrum mask, which is shown in Fig. 1 and Fig. 2 for indoor and outdoor environments, respectively ${ }^{[8]}$.

The above spectral mask allows UWB devices to overlay existing systems while ensuring sufficient attenuation to limit adjacent channel interference; i.e. the UWB effective isotropic radiated power(EIRP) emission level is restricted to $-41 \mathrm{dBm} / \mathrm{MHz}$ constant PSD over a 7.5 $\mathrm{GHz}$ bandwidth, which implies approximately $0.55 \mathrm{~mW}$ average transmission power. Additional PSD limits have been placed below $2 \mathrm{GHz}$ to protect critical applications such as global positioning system(GPS $)^{[9]}$. Because of the shape of this spectral mask, it needs to use additional transmission filtering of base-band pulses to limit the out-of-band emission spectra. Since the UWB spectrum has an unlicensed nature, all wireless devices sharing the spectrum must coexist. In other words, the interference should be kept as low as possible, regardless of present or future spectral allocations and emissions restrictions in various regions of the world ${ }^{[10],[11]}$. According to MBOA, multiband OFDM is capable of complying with local regulations by dynamically turning off certain tones or channels in software, a capability which speaks to their favor. However, it is still worth pointing out that the physical layer characteristics are not standardized yet. In summary, UWB communications are allowed to transmit signals with very low average transmission power compared to more conventional(narrow band) systems that effectively restricts UWB to short ranges. UWB is, thus, a candidate physical layer mechanism for the IEEE 802.15 wireless personal area network(PAN) for shortrange high-rate connectivity.

\section{2-2 ITS}

ITS technology has been developed to solve traffic problems such as traffic congestion and accidents and to reduce the overall congestion cost. The final goal of ITS will be to improve the traffic efficiency and mobile safety without new road construction. Dedicated shortrange communication(DSRC) ${ }^{[12]}$ provides a high-speed radio link between the road-side unit(RSU) and on-board unit(OBU) within the narrow communication area. ITSrelated information can be transferred based on packet frame within the communication area formed by the roadside antenna. DRSC communication will be a fundamental technology for ITS services. DSRC communication systems have been developed worldwide and recently applied for electronic toll collection(ETC). But most of all, ITS services will be provided by DSRC communication technology. There are two schemes in 
DSRC communication, which are active type and passive type.

The two most widely deployed DSRC applications are ETC and automatic equipment identification(AEI). ETC is the application of DSRC to simplify the payment of tolls. The DSRC link is used by the vehicle to provide account information to a toll facility. There are over one million electronic toll tags currently deployed in the US, with the majority of the tags manufactured by a few vendors. Each vendor tends to dominate a region of the country, essentially defining de facto regional ETC standards that are based upon specific product lines. To provide inter-regional interoperability, some vendors are building multi-mode devices that support more than just their own data link protocols. However, it is unclear whether this approach can lead to national interoperability due to a number of factors including cost and projected market demand. AEI, which uses the DSRC link to transmit an identification number to the roadside, increases freight transportation efficiency because it permits the automation of asset tracking and management. Since this capability has become critical for the commercial freight industry, especially with the advent of "just in time" delivery, motor and rail carriers have acquired over 3.5 million tags and attached them to tractors, trailers, containers, and railroad rolling stock. These tags typically follow AEI standards issued by the American Trucking Association, Association of American Railroads, International Standards Organization(ISO), or the American National Standards Institute(ANSI). Note that an AEI tag is different than tags used in the manufacturing and housing environments. Currently, the National Committee for Information Technology Standards(NCITS) Non-Contact Information Systems Interface (T6) Technical Committee is developing a standard for tags that can be placed on individual items, boxes, pallets, etc. This standard defines a link that will operate unlicensed in the $2.45 \mathrm{GHz}$ industrial, scientific and medical(ISM) band and has both a frequency- hopping and direct sequence spread spectrum mode. Although ETC and AEI are widely deployed, there are many other applications that will be based on DSRC. The US department of transportation federal highway administration(FHWA) has developed a national ITS architecture that has identified a number of other potential DSRC applications including:

- Parking management

- Traffic flow monitoring

- Intersection collision warning/avoidance

- In-vehicle signing(i.e., information usually conveyed by roadside signs is transmitted to the vehicle for internal display)
Table 1. Parameters of propagation path loss model.

\begin{tabular}{|c|c|c|c|c|}
\hline Environments & $\gamma$ & $P L_{0}[\mathrm{~dB}]$ & $d_{0}[\mathrm{~m}]$ & $\sigma[\mathrm{m}]$ \\
\hline $\begin{array}{c}\text { Indoor residential } \\
\text { LOS }\end{array}$ & -1.7 & $20 \log \left(4 \pi f d_{0} / c\right)$ & 1 & 1.5 \\
NLOS & $3.5 \sim 5$ & $20 \log \left(4 \pi f d_{0} / c\right)$ & 1 & $2.7 \sim 4$ \\
Hard NLOS & $\geqq 7$ & $20 \log \left(4 \pi f d_{0} / c\right)$ & 1 & 4 \\
\hline $\begin{array}{c}\text { Indoor office/ } \\
\text { laboratory }\end{array}$ & & & & \\
LOS & -1.5 & $20 \log \left(4 \pi f d_{0} / c\right)$ & 1 & $0.3 \sim 4$ \\
NLOS & $2 \sim 4$ & $20 \log \left(4 \pi f d_{0} / c\right)$ & 1 & $1.2 \sim 4$ \\
Hard NLOS & $4-7.5$ & $20 \log \left(4 \pi f d_{0} / c\right)$ & 1 & $\geqq 4$ \\
\hline $\begin{array}{c}\text { Outdoor rural/ } \\
\text { general }\end{array}$ & & & & \\
LOS & -2 & $20 \log \left(4 \pi f d_{0} / c\right)$ & 1 & $0.5 \sim 1$ \\
NLOS & $3 \sim 4$ & $20 \log \left(4 \pi f d_{0} / c\right)$ & 1 & $<3$ \\
\hline
\end{tabular}

- Automated highway system

- Emergency vehicle signal pre-emption

- Transit vehicle signal priority

- Commercial vehicle weigh station bypass

- Commercial vehicle international border crossing

Some of these applications can be supported by a single stand alone tag, while others may require integration with in-vehicle electronic databases, connections to on-board computers or interfaces to smart cards. The type of application will dictate the specific tag configuration. Thus, DSRC, either alone or in combination with other ITS technologies, could support a wide range of safety, travel efficiency, and traveller conveniencerelated services.

\section{Interference Analysis between UWB and ITS(DSRC)}

\section{3-1 Path Loss Model}

We used the UWB path loss model ${ }^{[13]}$. The path loss model based on extensive UWB indoor and outdoor experiments is given by

$$
P L(d)=P L_{0}\left(d_{0}\right)+10 \mathrm{\gamma} \log \left(\frac{d}{d_{0}}\right)+X_{0},
$$

where $P L(d)$ is the path loss, $P L_{0}\left(d_{0}\right)$ is the intercept point at distance $d_{0}, 10 \mathrm{\gamma} \log \left(\frac{d}{d_{0}}\right)$ is the media path loss reference to $d_{0}, \mathrm{\gamma}$ is referred to as the path loss exponent, and $X_{\sigma}$ is the lognormal shadow fading. The propagation path loss model parameters are given by Table 1.

\section{3-2 UWB Interference Model}

We consider the situation where ITS units are sur- 


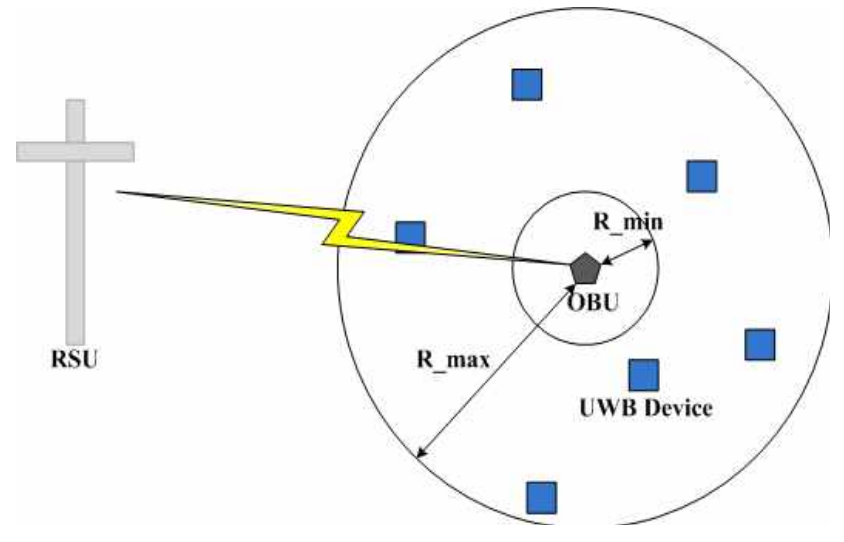

Fig. 3. Interference scenario.

rounded by a UWB device in a 2-dimensional setting. Fig. 3 shows the interference scenario.

The victim receiver is placed at the center of two circles. The inner circle defines the boundary of a UWBfree zone. In other words, UWB devices do not exist closer to the victim receiver. In between the inner and outer circles, the UWB devices are distributed uniformly over the surface. We describe the probability density function(pdf) of the UWB device as a function of the radius.

$$
f_{U W B}(r)=\left\{\begin{array}{cl}
0 & r<r_{\min }, r>r_{\max } \\
\frac{2 r}{r_{\text {max }}^{2}-r_{\min }^{2}} & r r_{\min } \leq r \leq r_{\max }
\end{array} .\right.
$$

Also, if the number of the interfering UWB devices is $N$, the density of UWB devices is

$$
\rho=\frac{N}{\pi\left(r_{\max }^{2}-r_{\min }^{2}\right)} .
$$

If a UWB device exists in the outer circle, the UWB interference power $P_{r}$ that is received from the ITS receiver is given by

$$
P_{r}(r)=2 \pi \rho P_{U W B}\left(\frac{\lambda}{4 \pi}\right)^{2} I\left(r_{\min }, d_{0}\right),
$$

where $\rho$ is the UWB density in user $/ \mathrm{m}^{2}, P_{U W B}$ is the transmission power for each UWB device, $\lambda$ is the wave length, and $I\left(r_{\min }, d_{0}\right)$ is a factor depending on the environment through $d_{0}$ and $r_{\min }$. We can write the total interference power for the UWB devices when the total UWB devices are in the area.

$$
P_{U W B}=\sum_{r=1}^{N} P_{r}
$$

From ITU Document $1-8 / 8$ E, the SINR at the victim receiver is defined by

$$
S I N R=\frac{P_{S} \cdot \text { Const }}{N_{0}+I_{\text {UWB }}},
$$

where $N_{0}$ is thermal noise, $P_{\text {ITS }}$ is the transmitter power at the ITS access point, Const is the path loss as a function of distance, and $I_{U W B}$ is the total perceived power from all the UWB devices around the victim receiver. Then, we get

$$
\begin{aligned}
\operatorname{SINR}(d B)= & P_{I T S}(d B m)+\operatorname{Con~st}(d B), \\
& -N(d B m)-M(d B) \\
\operatorname{SINR}(d B)= & S I N R_{\text {without UwB }}(d B)-M(d B),
\end{aligned}
$$

where we have defined $M$ such as

$$
\begin{aligned}
10 \log \left(\frac{N_{0}+I_{U W B}}{10^{-3}}\right) & =10 \log \left(\frac{N_{0}+I_{U W B}}{N_{0}} \times \frac{N_{0}}{10^{-3}}\right) \\
& =N_{0}(d B m)+M(d B)
\end{aligned}
$$

Therefore, any increase of $M \mathrm{~dB}$ due to UWB interference will result in an equal decrease in the SINR. Hence, if we assume that the system can support a degradation of $M \mathrm{~dB}$ in SINR, we can deduce the total amount of UWB interference corresponding to a $M \mathrm{~dB}$ degradation such that

$$
I_{U W B}=\left(N_{0} \times 10^{\frac{M(d B)}{10}}\right)-N_{0}=N_{0}\left(10^{\frac{M(d B)}{10}}-1\right) .
$$

We have also found a general value for the UWB interference as a function of the UWB transmitter density from $r_{\min }$ to infinity, which is given by

$$
I_{U W B}=2 \pi \rho P_{U W B}\left(\frac{\lambda}{4 \pi}\right)^{2} I\left(r_{\min }, d_{0}\right),
$$

where

$$
\begin{aligned}
& I\left(r_{\min }, d_{0}\right)= \\
& \quad \frac{\left(d_{0}+R_{\min }\right)\left(\ln \left(d_{0}+r_{\min }\right)-\ln \left(r_{\min }\right)\right)-d_{0}}{\left(d_{0}+r_{\min }\right)} .
\end{aligned}
$$

\section{3-3 Thermal Noise Power}

The thermal noise is generated by thermal agitation of electrons in a conductor. The noise power, in watts, is given by

$$
\text { Noise Floor }=k T B_{R X},
$$

where $k$ is Boltzman's constant $\left(1.38 \times 10^{-23} \mathrm{~W} / \mathrm{Hz} / \mathrm{K}\right)$ and $B_{R X}$ is the receiver bandwidth at a temperature $T$ in Kelvin. Thus, in $\mathrm{dBm}$, we have

$$
\text { Noise Floor }=10 \log \left(\frac{k T B_{R X}}{1 m W}\right) .
$$

Therefore, for ITS, the bandwidth is $10 \mathrm{MHz}$. If we consider the temperature of $T=290 \mathrm{~K}$, the noise floor is $-104 \mathrm{dBm}$. 


\section{3-4 UWB Emission Limit}

Table 2 specifies the average emission limits in terms of EIRP as measured with $1 \mathrm{MHz}$ resolution bandwidth that we are implementing for UWB operation ${ }^{[1]}$.

\section{3-5 Minimum Distance with a UWB Device for 1-dB De- gradation}

In order to represent the effect of interference from UWB on the ITS device, it is interesting to consider only one UWB transmitter and estimate the necessary minimum separation distance to degrade the signal by no more than $M \mathrm{~dB}$. If we consider $M \mathrm{~dB}$ degradation in the SNR, the maximum interference level is as follows.

$$
\begin{array}{r}
P_{T}-\left(P L_{0}\left(d_{0}\right)+10 \mathrm{\gamma} \log \left(\frac{d}{d_{0}}\right)+X_{\sigma}\right)+G_{R}, \\
\leq N_{0}\left(10^{\frac{M}{10}}-1\right)
\end{array}
$$

where $G_{R}$ is antenna gain of the receiver antenna. Therefore, we can find the minimum distance $d$, which satisfies (15).

\section{3-6 Maximum Possible UWB Emission Power for 1-dB Degradation}

If the system can tolerate a $1-\mathrm{dB}$ degradation in the SNR, the permitted received UWB interference at the victim node is given by

$$
I_{\text {UWB }}=N_{0}\left(10^{\frac{M}{10}}-1\right) \cong 0.25 \times N_{0},
$$

where $N_{0}$ is the thermal noise, which is equal to -110 $\mathrm{dBm}$ in the ITS receiver bandwidth $10 \mathrm{MHz}$. A 1-dB degradation in the SNIR corresponds to about a $20 \%$ decrease in the received SINR. So, we can estimate the $I_{U W B}$, which is given by

$$
I_{U W B}=-110 \mathrm{dBm} / \mathrm{MHz} \text {. }
$$

Therefore, we can find the maximum UWB emission power for 1-dB degradation in SINR, which is given by

$$
P_{T}=-110+\left(P L_{0}\left(d_{0}\right)+10 \mathrm{\gamma} \log \left(\frac{d}{d_{0}}\right)+X_{\circ}\right)-G_{R^{*}}
$$

\section{Numerical Results}

In Table 3, the minimum distance between UWB and ITS devices is shown. From Table 3, we know that if we only consider the NLOS situation because the LOS path between UWB and DMB-T devices seldom exists indoor and outdoor, coexistence between UWB and DMB$\mathrm{T}$ devices can be realized if the UWB device is separated at least $5 \mathrm{~m}$ indoor and $7 \mathrm{~m}$ outdoor from the ITS device.

In Fig. 4, the maximum UWB emission power in the case of down link of ITS in an indoor office environment is shown. It should be noted that an LOS path between the transmitter and receiver seldom exists in the indoor environment because of natural or man-made blocking and one must rely on the signal via multipath. It seems that when the LOS exists between the UWB transmitter and ITS receiver, the UWB device has a lower possible emission power than the NLOS situation

Table 3. Minimum distance between UWB and ITS devices.

\begin{tabular}{|l|c|}
\hline \multicolumn{1}{|c|}{ Situation } & Minimum distance $(\mathrm{m})$ \\
\hline Indoor LOS & 23 \\
\hline Indoor NLOS & 5 \\
\hline Outdoor LOS & 16 \\
\hline Outdoor NLOS & 7 \\
\hline
\end{tabular}

Table 2. Average emission limits applicable to UWB operation.

\begin{tabular}{|c|c|c|c|c|c|}
\hline Frequency band(MHz) & $\begin{array}{c}\text { Imaging below } \\
960 \mathrm{MHz}\end{array}$ & $\begin{array}{c}\text { Imaging mid } \\
\text { frequency }\end{array}$ & $\begin{array}{c}\text { Imaging high } \\
\text { frequency }\end{array}$ & $\begin{array}{c}\text { Indoor } \\
\text { application }\end{array}$ & $\begin{array}{c}\text { Vehicular } \\
\text { radar }\end{array}$ \\
\hline $0.009 \sim 960$ & -15.290 & -15.290 & -15.290 & -15.290 & -15.290 \\
\hline $960 \sim 1,610$ & -65.3 & -46.3 & -65.3 & -65.3 & -75.3 \\
\hline $1,610 \sim 1,990$ & -53.3 & -41.3 & -53.3 & -53.3 & -61.3 \\
\hline $1,990 \sim 3,100$ & -51.3 & -51.3 & -51.3 & -51.3 & -61.3 \\
\hline $3,100 \sim 10,600$ & -51.3 & -51.3 & -41.3 & -41.3 & -61.3 \\
\hline $10,600 \sim 22,000$ & -51.3 & -51.3 & -51.3 & -51.3 & -61.3 \\
\hline $22,000 \sim 29,000$ & -51.3 & -51.3 & -51.3 & -51.3 & -41.3 \\
\hline Above 29,000 & -51.3 & -51.3 & -51.3 & -51.3 & -51.3 \\
\hline
\end{tabular}




\section{Indoor office (Downlink)}

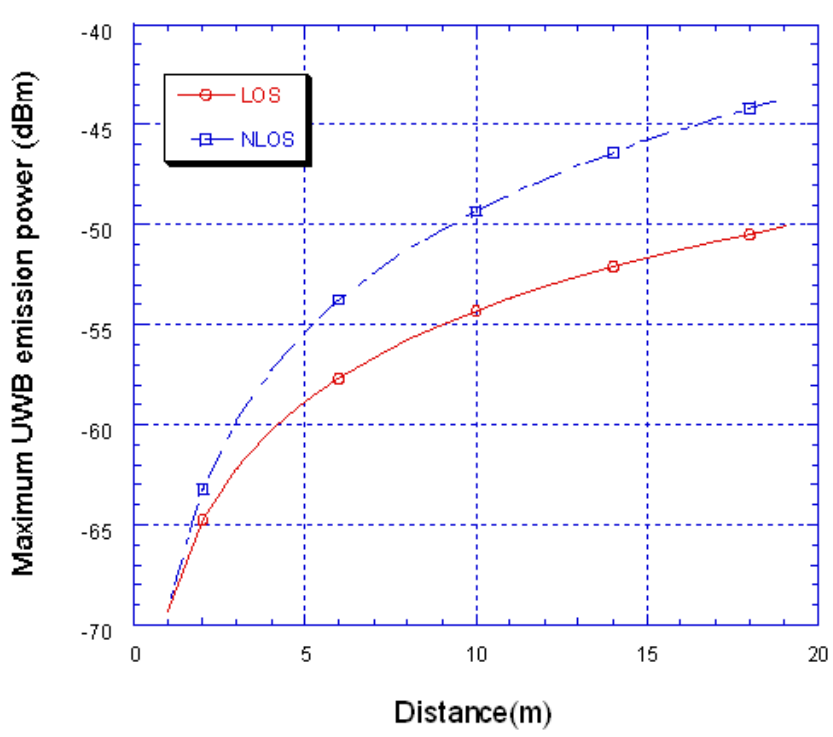

Fig. 4. Maximum possible UWB emission power in indoor office(downlink).

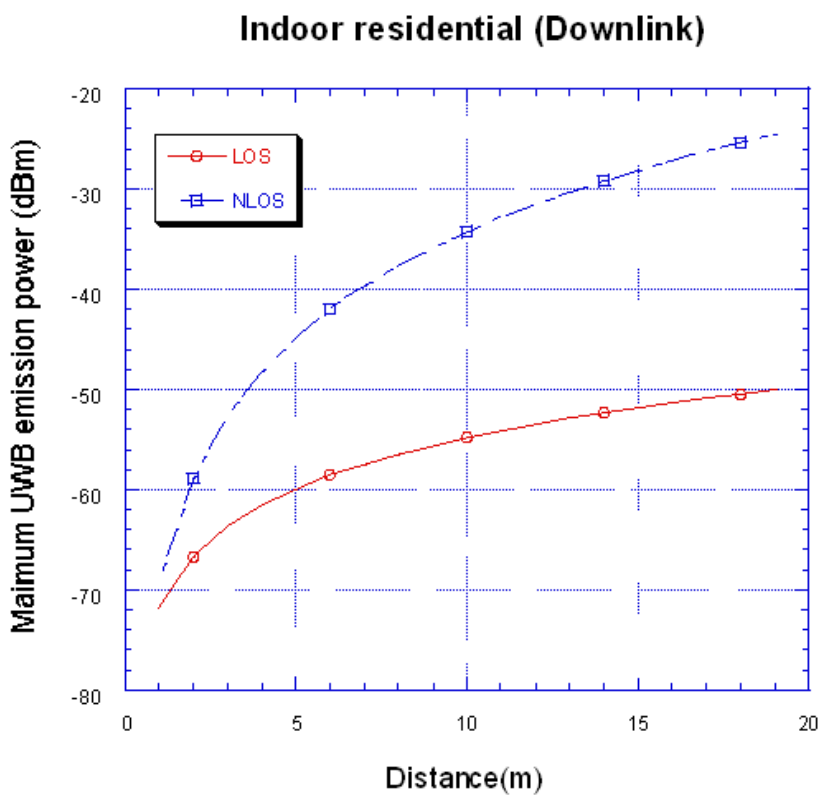

Fig. 5. Maximum possible UWB emission power in indoor residential(downlink).

due to difference in path loss.

In Fig. 5, the maximum UWB emission power in the case of down link of ITS depends on the distance in the indoor residential environment. It seems that when the LOS exists between the UWB transmitter and ITS receiver in the indoor residential environment, a UWB device also has a lower possible emission power than the NLOS situation due to difference in path loss.

From Fig. 4 and Fig. 5, it seems that in a residential environment, path loss of the UWB signal is higher in an office environment. It should be noted that the possible emission power of the residential NLOS situation is higher than the office NLOS situation. This means that a UWB device is more adaptable in the residential environment than in the office environment. But in the LOS situation, the possible emission power of the UWB device in residential and office environment is not significantly different.

In Fig. 6, the maximum UWB emission power in the case of down link of ITS depends distance in the outdoor rural environment. It seems that the LOS exists between the UWB transmitter and ITS receiver, and the UWB device has a lower possible emission power than the NLOS situation due to difference in path loss.

In Fig. 7, the maximum UWB emission power in the case of uplink of ITS in the indoor office environment is shown. It should be noted that an LOS path between the transmitter and receiver seldom exists in the indoor environment because of natural or man-made blocking and one must rely on the signal via multipath. It seems that when the LOS exists between the UWB transmitter and ITS receiver, the UWB device has a lower possible emission power than the NLOS situation due to difference in path loss.

In Fig. 8, the maximum UWB emission power in the case of up link of ITS depends on distance in the indoor residential environment. It seems that when the LOS exists between a UWB transmitter and ITS receiver in the indoor residential environment, the UWB device also has a lower possible emission power than the NLOS situation due to difference in path loss.

\section{Outdoor rural (D ownlink)}

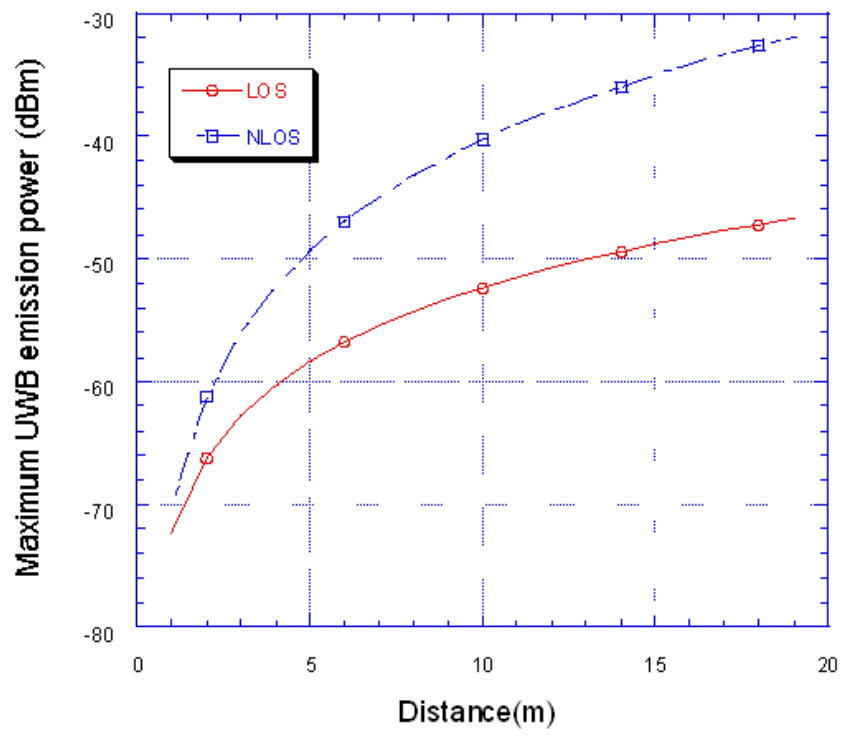

Fig. 6. Maximum possible UWB emission power in outdoor rural(downlink). 


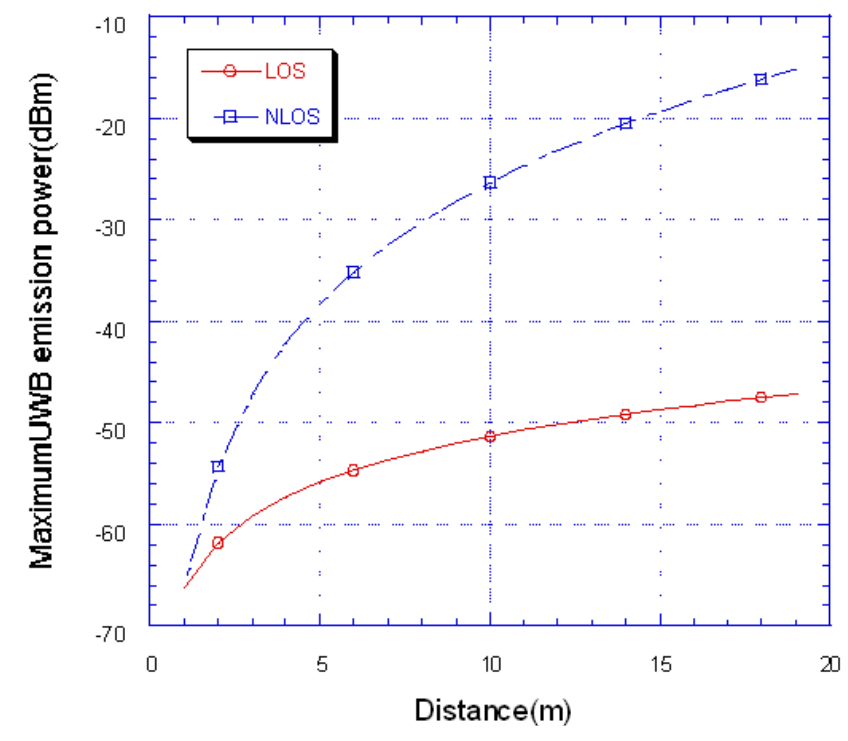

Fig. 7. Maximum possible UWB emission power in indoor office(uplink).

\section{Indoor residential (Uplink)}

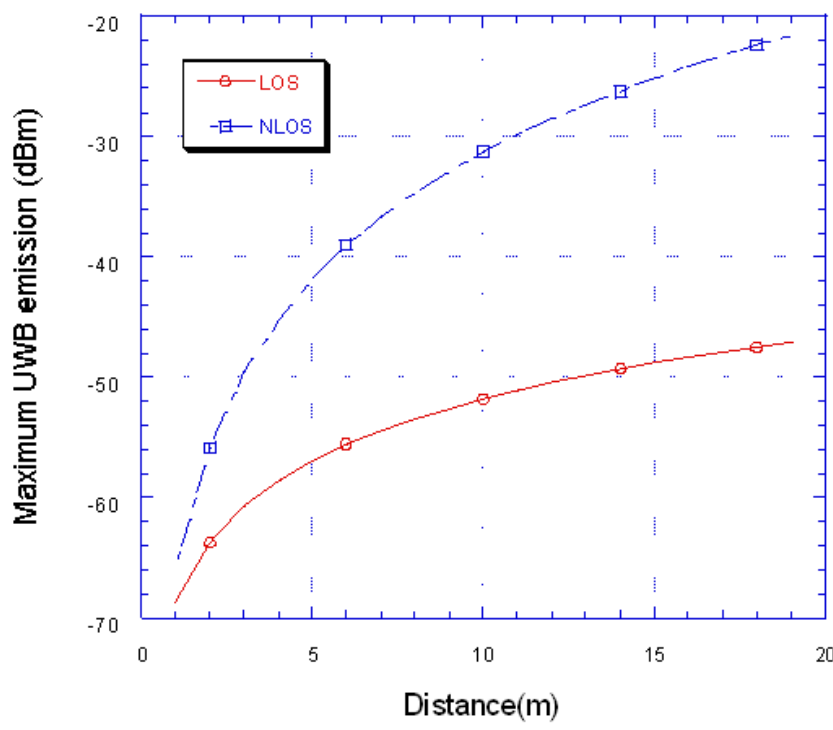

Fig. 8. Maximum possible UWB emission power in indoor residential(uplink).

From Fig. 7 and Fig. 8, it seems that in a residential environment, the UWB device has a higher possible emission power than in the office environment because in a residential environment, path loss of the UWB signal is higher than in the office environment. It should be noted that the possible emission power of the residential NLOS situation is higher than that of the office NLOS situation. This means that the UWB device is more adaptable in a residential environment than in an office environment. But in the LOS situation, the possible emission power of the UWB device in residential

\section{Outdoor rural (Uplink)}

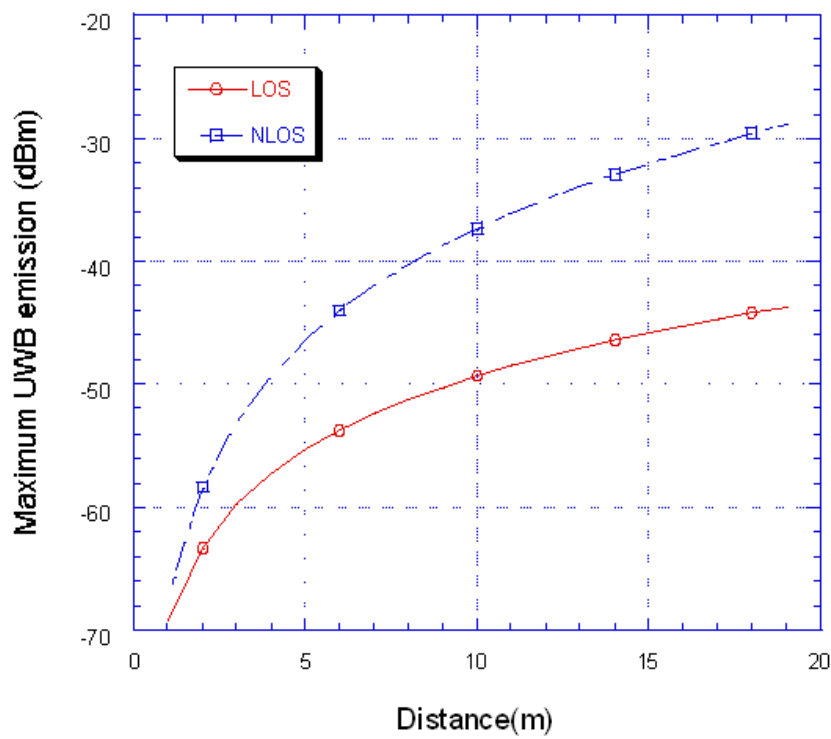

Fig. 9. Maximum possible UWB emission power in outdoor(uplink).

and office environments is not significantly different.

In Fig. 9, the maximum UWB emission power in the case of up link of ITS depends on distance in the outdoor rural environment. It seems that the LOS exists between the UWB transmitter and ITS receiver, and the UWB device has a lower possible emission power than the NLOS situation due to difference in path loss.

From Fig. 3 to Fig. 8, we can obtain the results in Table 4 and Table 5. We can compare the results with the FCC mask using Table 6. Thus, coexistence of UWB and ITS devices can be realized if the emission mask is at least $19.3 \mathrm{~dB}$ for indoor application or $19.3 \mathrm{~dB}$ for image system, bellow the current FCC limit in indoor environments. And in outdoor settings, coexistence of UWB and DMB-T devices can be realized if the emission mask is $1.6 \mathrm{~dB}$ over the current FCC limit in outdoor environments.

Table 4. Maximum possible UWB emission power(down link).

\begin{tabular}{|l|c|c|}
\hline \multicolumn{1}{|c|}{ Situation } & $\begin{array}{c}\text { Distance } \\
(\mathrm{m})\end{array}$ & $\begin{array}{c}\text { Emission } \\
\text { limit }(\mathrm{dBm})\end{array}$ \\
\hline Indoor office LOS & 3 & -62.1 \\
\hline Indoor office NLOS & 3 & -59.7 \\
\hline Indoor residential LOS & 3 & -63.6 \\
\hline Indoor residential NLOS & 3 & -51.6 \\
\hline Outdoor rural LOS & 3 & -62.7 \\
\hline Outdoor rural NLOS & 3 & -52.9 \\
\hline
\end{tabular}


Table

5. Maximum possible UWB emission power(uplink).

\begin{tabular}{|l|c|c|}
\hline \multicolumn{1}{|c|}{ Situation } & $\begin{array}{c}\text { Distance } \\
(\mathrm{m})\end{array}$ & $\begin{array}{c}\text { Emission } \\
\text { limit(dBm) }\end{array}$ \\
\hline Indoor office LOS & 3 & -60.6 \\
\hline Indoor office NLOS & 3 & -49.6 \\
\hline Indoor residential LOS & 3 & -60.6 \\
\hline Indoor residential NLOS & 3 & -48.6 \\
\hline Outdoor rural LOS & 3 & -59.7 \\
\hline Outdoor rural NLOS & 3 & -52.9 \\
\hline
\end{tabular}

Table 6. Comparison of FCC mask and the results.

\begin{tabular}{|l|c|c|c|}
\hline \multicolumn{1}{|c|}{ UWB system } & $\begin{array}{c}\text { FCC } \\
\text { mask }\end{array}$ & Results & Difference \\
\hline Imaging system & -41.3 & -60.6 & -19.3 \\
\hline $\begin{array}{l}\text { Indoor application } \\
\text { (first order) }\end{array}$ & -41.3 & -60.6 & -19.3 \\
\hline Vehicles radar system & -61.3 & -59.7 & +1.6 \\
\hline
\end{tabular}

\section{Conclusions}

In this paper, the effect of interference between UWB system and ITS is analyzed. The maximum possible UWB emission power and minimum possible distance between UWB device and ITS is found. The minimum possible distance is $5 \mathrm{~m}$ indoors and $7 \mathrm{~m}$ outdoors from the ITS device. Coexistence of UWB and ITS devices can be realized if the emission mask is at least $19.3 \mathrm{~dB}$ for indoor application or $19.3 \mathrm{~dB}$ for image system bellow the current FCC limit in an indoor environment. And outdoors, coexistence of UWB and ITS devices can be realized if the emission mask is at least $1.6 \mathrm{~dB}$ over the current FCC limit for vehicles' radar systems in outdoor environments.

This paper can be used for standardization of not only UWB, but also other radio communication systems, and this interference analysis method can also be used for other radio communication systems.

This research was supported by the MKE(The Ministry of Knowledge Economy), Korea, under the ITRC(Information Technology Research Center) support program supervised by the NIPA(National IT Industry Promotion Agency(NIPA-2009-(C1090-09020005)).

\section{References}

[1] "First report and order in the matter of revision of Part 15 of the commission's rules regarding ultrawideband transmission systems", FCC, Released, ET Docket 98-153, FCC 02-48, Apr. 2002.

[2] D. Landi, C. Fischer, "The effects of UWB interference on GSM systems", in Proc. of Int. Zurich Seminar, pp. 86-89, Feb. 2004.

[3] "UWB interference to IEEE 802.11A/B WLAN", ITU Document 1-8/8-E, Oct. 2003.

[4] "Study of interference effects of a UWB mass deployment on GSM systems", ITU Document, 1-8/9-E, Oct. 2003.

[5] "UWB interference with Bluetooth", ITU Document 1-8/11-E, Oct. 2003.

[6] "Monte-Carlo simulation methodology for the use in sharing and compatibility studies between different radio service or system", ERC Report 68, Jun. 2002.

[7] C. L. Bennett, G. F. Ross "Time-domain electromagnetics and its applications", in Proc. of IEEE, vol. 66, pp. 299-318, Mar. 1978.

[8] "FCC notice of proposed rule making, revision of part 15 of the commission's rules regarding ultra wideband transmission systems", FCC, Washington, DC, ET-Docket 98-153.

[9] M. Hamalainen, V. Hovinen, R. Tesi, J. H. J. Iinatti, and L. Matti, "On the UWB system coexistence with GSM900, UMTS/WCDMA, and GPS", IEEE $J$. Select. Areas Commun., vol. 20, no. 9, Dec. 2002.

[10] J. Huschke, "ANNEX 5-12, cellular mobile networks", M18_30R0_SE24,CEPT SE24 M18, Copenhagen, Dec. 2002.

[11] S. Plimmer, D. Barker, "Impact of UWB on thirdgeneration telecommunications(3G)-final report", M19_50R0_SE24_UWB_UK, CEPT SE24 M19, Copenhagen, Mar. 2003.

[12] H. Chang, Y. Kim, "A study on DSRC technical standards for ITS systems", available at http:// www.itsgis.net.

[13] "UWB Path Loss Models", ITU Document 1-8/6E, Document 3k/5E, Document 3M/4-E, Oct. 2003. 


\section{Se ho Park}

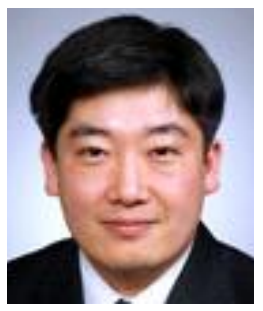

received his B.S. and M.S. degrees from the School of Electrical Engineering, Seoul National University, Korea in 1997 and 1999 respectively. He joined several research projects on the wireline/ wireless modem design at the Institute of New Media and Communications(INMC), Seoul National University from 1999 to 2001. In 2001, He joined the Network Division of SK Telecom, the first commercial CDMA operator in the world. He has worked at network/platform/terminal R\&D sections and currently is working at the network evolution strategy part of the Network Planning Office, SK Telecom. In this company, he has experienced several commercial technologies based on IS-95A/B, EV-DO, WCDMA, HSPA networks and mobile terminals. He is also a Ph.D candidate student of the School of Electronics Engineering, Kwangwoon University, Seoul, Korea. His main research interests are design and implementation of wireless communication systems for next generation commercial mobile communication services and cognitive radio.

\section{Eun Cheol Kim}

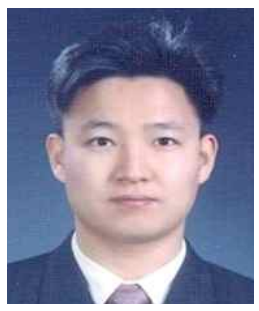

received the B.S. and M.S. degrees from the Department of Wireless Communications Engineering, Kwangwoon University, Seoul, Korea, in 2003 and 2005, respectively. His research interests include next generation communication systems and their applications such as code division multiple access(CDMA), ultrawide band(UWB), orthogonal frequency division multiplexing OFDM), multiple-input multiple-output(MIMO), cognitive radio(CR), chip synchronization, network synchronization, jitter rejection, channel coding, and compatibility analysis between radio communication services. He received the Best Paper Award published by the IEEK(2008). He is currently working toward the Ph.D. degrees at Kwangwoon University, Seoul, Korea. He is a Member of IEEE, IEEK, KICS, and IWIT.

\section{Jin Young Kim}

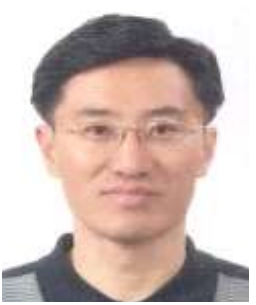

He received the B. Sc., M. Sc., and Ph. D. degrees from the School of Electrical Engineering, Seoul National University (SNU), Seoul, Korea, in 1991, 1993, and 1998, respectively. He was Member of Research Staff at the Institute of New Media and Communications(INMC) and at the Inter-university Semiconductor Research Center(ISRC) of the SNU from 1994 to 1998. He was Postdoctoral Research Fellow at the Department of Electrical Engineering, Princeton University, NJ, U.S.A, from 1998 to 2000. He was Principal Member of Technical Staff at the Central Research and Development Center, SK Telecom, Korea, from 2000 to 2001. He is currently Associate Professor at the School of Electronics Engineering, Kwangwoon University, Seoul, Korea. Now, he has his sabbatical leave as Visiting Scientist at the LIDS(Laboratory of Information and Decision Systems), Massachusetts Institute of Technology M.I.T), MA, U.S.A. His research interests include design and implementation of wireline/wireless multimedia communication systems for applications to spread-spectrum, cognitive radio, ultrawideband(UWB), space communication, optical communication and powerline communication systems with basis on modulation/demodulation, synchronization, channel coding, and detection/estimation theory. He received the Best Paper Awards from several academic conferences and societies including Jack Nebauer Best Systems Paper Awardfrom IEEE VT Society(2001), the Award of Ministry of Information and Communication of Korea Government(1998), the Best Paper Award at APCC'00(2000), the Best Paper Award at IEEE MoMuC'97(1997), and the many other Best Paper Awards from conferences of IEEK'08, KITFE'08, KITS'08, and KITS'09(2008-2009). He was listed in the Marquis Who's Who in the World, Marquis Who's Who in Science and Engineering, ABI and IBC throughout from 2001 to 2009 Editions. He is now Senior Member of IEEE, Regular Member of IET, IEICE, and Life Member of IEEK, KICS, KEES, KITFE, KITS and KOSBE. 